Cite this: Phys. Chem. Chem. Phys., 2013, 15, 4995

Received 29th November 2012, Accepted 1st February 2013

DOI: $10.1039 / \mathrm{c} 3 \mathrm{cp} 44278 \mathrm{k}$

www.rsc.org/pccp

\section{Anisotropy of the water-carbon interaction: molecular simulations of water in low-diameter carbon nanotubes}

\author{
Guillermo Pérez-Hernández* and Burkhard Schmidt
}

\begin{abstract}
Effective Lennard-Jones models for the water-carbon interaction are derived from existing high-level $a b$ initio calculations of water adsorbed on graphene models. The resulting potential energy well $\varepsilon_{\mathrm{CO}}+$ $2 \varepsilon_{\mathrm{CH}} \approx 1 \mathrm{~kJ} \mathrm{~mol}^{-1}$ ) is deeper than most of the previously used values in the literature on water in carbon nanotubes (CNTs). Moreover, a substantial anisotropy of the water-carbon interaction $\left(\varepsilon_{\mathrm{CO}} \approx\right.$ $2 \varepsilon_{\mathrm{CH}}$ ) is obtained, which is neglected in most of the literature. We systematically investigate the effect of this anisotropy on structure and dynamics of TIP5P water confined in narrow, single-walled CNTs by means of molecular dynamics simulations for $T=300 \mathrm{~K}$. While for isotropic models water usually forms one-dimensional, ordered chains inside $(6,6)$ CNTs, we find frequent chain ruptures in simulations with medium to strongly anisotropic potentials. Here, the water molecules tend to form denser clusters displaying a liquid-like behaviour, allowing for self-diffusion along the CNT axis, in contrast to all previous simulations employing spherical $\left(\varepsilon_{\mathrm{CH}}=0\right)$ interaction models. For $(7,7)$ CNTs we observe structures close to trigonal, helical ice nanotubes which exhibit a non-monotonous dependence on the anisotropy of the water-carbon interaction. Both for vanishing and for large values of $\varepsilon_{\mathrm{CH}}$ we find increased fluctuations leading to a more liquid-like behaviour, with enhanced axial diffusion. In contrast, structure and dynamics of water inside $(8,8)$ CNTs are found to be almost independent of the anisotropy of the underlying potential, which is attributed to the higher stability of the non-helical fivefold water prisms. We predict this situation to also prevail for larger CNTs, as the influence of the water-water interaction dominates over that of the water-carbon interaction.
\end{abstract}

\section{Introduction}

Single walled carbon nanotubes (CNTs) can be considered as (chiral or achiral) rolls from a sheet of graphene, the "mother of all graphitic materials". ${ }^{1}$ Since their first discovery in $1991,{ }^{2}$ the study of CNTs has become an extremely active field in chemistry, physics and nanotechnology. ${ }^{3,4}$ A central issue in many of the (present or potential) applications of CNTs is the possibility to store or convey fluids. Among many others, the transport of water through CNTs may be of importance for filtering techniques ${ }^{5}$ and it is also considered as a simplified model for water channels in cell membranes. ${ }^{6}$ Since microscopically detailed experiments on molecular structures ${ }^{7}$ or on flow dynamics ${ }^{8,9}$ in CNTs are still rather scarce, most studies of these systems are based on molecular dynamics (MD) simulations. ${ }^{10}$ In addition to investigations of superlubricity, i.e., the enhanced flow of water through

Institut für Mathematik, Freie Universität Berlin, Arnimallee 6, D-14195 Berlin, Germany.E-mail: guille.perez@fu-berlin.de
CNTs ${ }^{11-16}$ another branch of MD studies focuses on the understanding of structures and hydrogen-bonded (H-bonding) networks of water molecules inside CNTs. Being confined in tubes with diameters not much larger than their own size, the water molecules adopt hydrogen-bonded structures distinctly different from those of bulk water. One of the narrowest CNTs known to be water-permeable are $(6,6)$ CNTs with a diameter of $0.81 \mathrm{~nm}$. There, the water molecules were found to arrange in a quasi onedimensional ordered chain. ${ }^{11,17-20}$ From $(7,7)$ CNTs (diameter $0.94 \mathrm{~nm}$ ) onwards, the water molecules tend to cover the inner CNT walls, typically showing layered structures which, upon freezing, can form so-called ice nanotubes (INTs). Simulation studies show that, in close analogy to the surrounding CNTs, also INTs can be found as chiral forms, i.e., water helices ${ }^{21-24}$ or as achiral forms, i.e. stacked water polygons. ${ }^{10,22,24-26}$ All of these modifications can be regarded as new phases of water, ${ }^{27}$ and their dependence on temperature, pressure or other simulation details has been investigated, e.g., in ref. 24, 28-30, aiming at establishing phase diagrams for water confined in CNTs. 
Because of the large system sizes in studies of water in CNTs, practically all of the above-mentioned simulation studies are based on empirical force fields. In particular, pairwise LennardJones (LJ) potential models with empirical parameters are used. Such LJ-models are implemented in most of the widely-used force fields and MD program packages, e.g., the OPLS-AA force field $^{31,32}$ implemented in the GROMACS program package. ${ }^{33-35}$ Hence, it is a major challenge to obtain quantitatively correct parameters for these models. On the one hand, experimental results suitable for the fitting of these parameters are hardly available. ${ }^{36}$ On the other hand, $a b$ initio calculations of water in CNTs are not computationally affordable, while standard density functional theory (DFT) calculations of water in CNTs are somewhat unreliable, due to problems with describing the van der Waals (vdW) dispersion interaction quantitatively. ${ }^{37}$ However, in recent years the interaction of a single water molecule with graphene has emerged as a benchmark system for high-level quantum chemistry methods for systems dominated by vdW interaction. ${ }^{37-42}$ In the present work, we use these existing results to reparametrize $\mathrm{LJ}$ models for the interaction between water and carbon-based materials. Since the quantum-chemical data show a strong dependence of the water-graphene interaction on the relative orientation of the two entities, particular emphasis shall be put on extracting the anisotropy of the water-carbon $\mathrm{LJ}$ model interaction as well. To the best of our knowledge, a similar approach has only been pursued in ref. 43 where rather strongly anisotropic LJ potential models were obtained by fitting the respective parameters to DFT calculations, however, only for water outside CNTs, where the curvature of the carbon layer is reversed.

In addition to the determination of anisotropic LJ models, the present work deals also with the influence of the respective model parameters on simulation results for the structure and dynamics of water in small diameter CNTs. Rather surprisingly, although these systems have been under investigation for more than 10 years now, not much systematic knowledge is available about the effect of the water-carbon interaction parameters. Among the few studies addressing this question is the review article of ref. 10 which compiles results of different MD studies for different temperatures and pressures as well as different water models. There, it is shown that rather small changes of $\mathrm{LJ}$ parameters can have strong effects on the structures of water molecules, e.g., the occurrence of water helices, stacked water polygons, or disordered structures. Also the flow dynamics of water in CNTs is known to depend on the interaction parameters, $c f$. the numerical filling experiments of ref. 11, where qualitative differences of the water occupancies of small CNTs were found for different LJ parameters of the water-carbon interaction. In a recent study, a more systematic investigation of the influence of the interaction strength between CNT wall and water can be found. ${ }^{15}$ There, both the water occupancy $\left(N_{\mathrm{H}_{2} \mathrm{O}}\right)$ and the water permeability of low diameter CNTs are shown to depend in a nearly stepwise fashion on the watercarbon LJ well depth. Notable wetting of CNT inner walls as well as flux through them are only observed if the well depth exceeds a certain threshold beyond which saturation effects occur. However, these studies, as well as most of the earlier MD studies, are based on the assumption of an isotropic water-carbon interaction, i.e. they neglect the effect of the water orientation on the interaction with carbon layers. Hence, the present work aims at filling this gap by providing a systematic investigation of anisotropic interaction models. In particular, we strive at varying the anisotropy while keeping the overall interaction strength of a single water molecule with a single carbon atom unchanged. As will be shown below, the anisotropy of the interaction with the confining CNT walls indeed has a substantial influence on certain structural and dynamical properties of the confined molecules, as has also been demonstrated in a very recent study of molecular hydrogen confined in CNTs. ${ }^{44}$

\section{Interaction potentials}

\section{A. Empirical Lennard-Jones models}

In typical MD simulations the total interaction energy between water and carbon-based materials such as graphene or CNTs is modelled by a pairwise sum of Lennard-Jones (LJ) potentials of the form

$$
V_{\mathrm{LJ}}(r)=4 \sum_{\mathrm{i}<\mathrm{j}} \varepsilon_{\mathrm{ij}}\left[\left(\frac{\sigma_{\mathrm{ij}}}{r_{\mathrm{ij}}}\right)^{12}-\left(\frac{\sigma_{\mathrm{ij}}}{r_{\mathrm{ij}}}\right)^{6}\right],
$$

where the sum extends over all nonbonded interactions between atoms $\mathrm{i}$ and $\mathrm{j}$ at distance $r_{\mathrm{ij}}$ and where $\varepsilon_{\mathrm{ij}}$ is the attractive well depth and $\sigma_{\mathrm{ij}}$ is the distance at which the pair potential is zero. For illustration, in the upper left part of Table 1, we list LJ-parameters used in previous studies of structure and dynamics of water in CNTs, similar to the compilation in Table 1 of ref. 36 . In many simulations, the carbon-water interaction is assumed to be isotropic (C-O interaction only, rows \# 1-3). Only in few simulations the anisotropy is modelled by additional LJ terms for the $\mathrm{C}-\mathrm{H}$ interaction (e.g. rows \# 4 and 5). In order to assess the models with and without $\mathrm{C}-\mathrm{H}$ interactions on an equal footing, as well as to allow for the reparametrization introduced below, we define the overall $(\eta)$ and anisotropic $(\delta)$ watercarbon interaction strengths as

$$
\eta=\varepsilon_{\mathrm{CO}}+2 \varepsilon_{\mathrm{CH}}, \quad \delta=1-\left(\varepsilon_{\mathrm{CO}}-2 \varepsilon_{\mathrm{CH}}\right) / \eta,
$$

while for the back transformation of our parameters to the original LJ-form we have

$$
\varepsilon_{\mathrm{CO}}=\eta(1-\delta / 2), \quad \varepsilon_{\mathrm{CH}}=\eta \delta / 4 .
$$

The overall well depth $\eta$ of water-carbon interaction is defined as the sum of the dispersion attractions of all three water atoms with carbon, hence describing the interaction of a carbon atom with a water molecule with all atom-atom pair distances at the respective minimum energy distances $(\sqrt[6]{2} \sigma)$. As can be seen in the upper left part of Table 1, this parameter varies strongly in the previously used models, with lowest and highest values differing by a factor of more than three. The anisotropy of the interaction energy strength is characterized by parameter $\delta$. In the isotropic limit $(\delta=0)$ there is no LJ interaction for hydrogen atoms $\left(\varepsilon_{\mathrm{CH}}=0\right)$, as in the case of most of the previous simulations which implicitly assume all of the dispersion interaction (all of the polarizability) to be located on 
Table 1 Energy $(\eta, \delta, \varepsilon)$ and range $(\sigma)$ parameters for water-carbon LennardJones interaction models of eqn (2), and resulting water-graphene minimum energies $\Delta E$ for down (d) and up (u) orientation of water (Fig. 1(a)), adsorbed on top of atoms (A), bonds (B) or centers (C) of the hexagons of the carbon lattice. Rows \# 1-5: sample empirical parameters from previous studies, partly adapted from Table 1 of ref. 36. Rows \# 6-9: fits to SCS-MP2 ${ }^{38}$ and $\operatorname{CCSD}(T)^{37}$ ab initio calculations. Energies $\Delta E, \eta, \varepsilon$ in $\mathrm{kJ} \mathrm{mol}^{-1}$, ranges $\sigma$ in $\mathrm{nm}$, anisotropy $\delta$ is dimensionless. The adsorption energies given in the right part of rows \# $1-5$ are obtained by extrapolating coronene molecules of increasing size

\begin{tabular}{lllllllllll}
\hline$\#$ & $\eta$ & $\delta$ & $\varepsilon_{\mathrm{CO}}$ & $\varepsilon_{\mathrm{CH}}$ & $\sigma_{\mathrm{CO}}$ & $\sigma_{\mathrm{CH}}$ & $\Delta E(\mathrm{~d})$ & $\Delta E(\mathrm{u})$ & Site & Ref. \\
\hline 1 & 0.314 & 0 & 0.314 & - & 0.319 & - & -4.5 & & $\mathrm{C}$ & 45 \\
2 & 0.392 & 0 & 0.392 & - & 0.319 & - & -5.6 & & $\mathrm{C}$ & 36 \\
3 & 0.478 & 0 & 0.478 & - & 0.328 & - & -7.2 & & $\mathrm{C}$ & 11 \\
4 & 0.647 & 0.798 & 0.389 & 0.129 & 0.328 & 0.281 & -8.6 & -7.1 & $\mathrm{C}$ & 46 \\
5 & 1.224 & 1.055 & 0.578 & 0.323 & 0.330 & 0.258 & -14.7 & -10.7 & $\mathrm{C}$ & 21 \\
6 & 1.117 & 1.640 & 0.201 & 0.458 & 0.328 & 0.277 & -13.0 & -8.2 & $\mathrm{C}$ & 38 \\
7 & 1.138 & 0.921 & 0.614 & 0.262 & 0.310 & 0.278 & -13.0 & -10.6 & $\mathrm{~A}$ & 37 \\
8 & 0.955 & 0.691 & 0.625 & 0.165 & 0.308 & 0.280 & -11.4 & -09.8 & $\mathrm{~B}$ & 37 \\
9 & 0.939 & 0.639 & 0.639 & 0.150 & 0.316 & 0.272 & -11.9 & -10.4 & $\mathrm{C}$ & 37
\end{tabular}

the oxygen atom. For $\delta>0$, the dispersive interaction of the $\mathrm{H}$ atoms is taken into account. For example, the values listed in rows \# 4 and 5 of Table 1 approach unity, corresponding to equal contributions of the $\mathrm{O}$-atom and the two $\mathrm{H}$-atoms to the dispersion interaction, i.e., $\varepsilon_{\mathrm{CO}} \approx 2 \varepsilon_{\mathrm{CH}}$. In contrast to the energy parameters $\eta$ and $\delta$, it can be seen in Table 1 that there is less uncertainty in the range parameters $\sigma_{\mathrm{CO}}$ and $\sigma_{\mathrm{CH}}$ of the LJ models found in the literature. Basically, the parameters are very close to the sums of the respective van der Waals radii of $\mathrm{C}$ $(0.170 \mathrm{~nm}), \mathrm{O}(0.152 \mathrm{~nm})$, and $\mathrm{H}(0.120 \mathrm{~mm}) .{ }^{47}$

Next, effective potential energy curves for the interaction of water with the benchmark system $\mathrm{C}_{58}$ (a coronene-like molecule used as a converged model for graphene, e.g. in ref. 37) are obtained by summing up all pairwise water-carbon interactions of eqn (1). The effective potentials vary drastically, with well depths $\Delta E$ ranging from 5 to $15 \mathrm{~kJ} \mathrm{~mol}^{-1}$ ( $c f$. Table 1), owing to the different $\eta$ values of the empirical models \# 1-5. In contrast, the zero crossings of the effective potentials show very little variations, being located between $0.26 \mathrm{~nm}$ and $0.28 \mathrm{~nm}$ in all cases. As expected, the anisotropic potential models \#4 and \# 5 show notable energetic differences between energies for the water down, i.e., for the hydrogen atoms pointing toward the graphene plane, and the reversed water up orientation, see Fig. 1(a). This difference is particularly large for LJ-model \#5 with $\delta \approx 1$, where a rotation of the water molecule from up to down leads to a stabilization energy of about four $\mathrm{kJ} \mathrm{mol}^{-1}$ at the equilibrium distance.

\section{B. Recalibration of $\mathrm{LJ}$ potential parameters}

To circumvent the uncertainties of previously used empirical models for the water-carbon interaction while keeping the simplicity and versatility of the pairwise additive LJ potentials, we fit the parameters $\eta, \delta, \sigma_{\mathrm{CO}}, \sigma_{\mathrm{CH}}$ to existing high-level quantum chemical calculations. Because no such data are available for the interaction of water with carbon nanotubes (one exception being the vdW-DFT study of water outside $\mathrm{CNTs}^{43}$ ), here we resort to data for the interaction of water with graphene. Now, we briefly review the most recent high level quantum chemistry results for the physisorption of water on graphene. Using DF-DFT-SAPT methods, Jordan et al. found a minimum energy of $\Delta E(\mathrm{~d})=-12.5 \mathrm{~kJ} \mathrm{~mol}^{-1}$ for water down orientation. $^{39,40}$ A similar value of $\Delta E(\mathrm{~d})=-13 \mathrm{~kJ} \mathrm{~mol}^{-1}$ was also obtained by Bludský et al. using DFT-CC methods ${ }^{41,42}$ as well as by Cabaleiro-Lago et al. using SCS-MP2 methods. The latter authors calculated a substantially weaker interaction $\Delta E(\mathrm{u})=-8 \mathrm{~kJ} \mathrm{~mol}^{-1}$ for the water $u p$ configuration. ${ }^{38}$ The effect of anisotropy was also investigated by Paulus et al. employing state of the art $\operatorname{CCSD}(\mathrm{T})$ methods with a triple zeta basis set. For a water molecule placed on top of a carbon atom of graphene (site $\mathrm{A})$, values of $\Delta E(\mathrm{~d})=-13.0$ and $\Delta E(\mathrm{u})=-10.6 \mathrm{~kJ} \mathrm{~mol}^{-1}$ were obtained, ${ }^{37}$ whereas the interactions of water located on top of a bond (site B) or a center of a hexagon (site C) are slightly weaker and less anisotropic. All these quantum chemical values of $\Delta E$ suggest that most of the previously used empirical water-carbon potentials are considerably too weak (e.g. \# 1-4), which is in coincidence with latest results for water outside a CNT. ${ }^{43}$ Only the LJ model \# 5 of ref. 21 yields a water-graphene attraction beyond $10 \mathrm{~kJ} \mathrm{~mol}^{-1}$, thus coming closest to the quantum chemical results. However, the energetic difference between down and up for LJ \# 5 appears to be slightly too large, whereas that difference is more reasonable for LJ model \# 4 from ref. 46.

Now, the quantum chemical values for the well depths, $\Delta E(\mathrm{~d})$ and $\Delta E(\mathrm{u})$, along with the corresponding positions of those minima, are used to obtain new LJ parameters $\eta, \delta, \sigma_{\mathrm{CO}}$, $\sigma_{\mathrm{CH}}$ by fitting a sum of pairwise additive LJ potentials (eqn (1)) to reproduce these minima as closely as possible. The resulting parameters are listed in the lower left part of Table 1. The range parameters $\sigma_{\mathrm{CO}}$ and $\sigma_{\mathrm{CH}}$ are again very close to the van der Waals values of $0.322 \mathrm{~nm}$ and $0.272 \mathrm{~nm},{ }^{47}$ respectively. While the overall interaction strength $\eta$ is found to be in a small interval of $[0.9,1.1] \mathrm{kJ} \mathrm{mol}^{-1}$, the results for the corresponding anisotropy $\delta$ are intriguing: the SCS-MP2 data of ref. 38 suggest a value of $\delta>1.6$ which appears somewhat counterintuitive, because it would imply stronger $\mathrm{C}-\mathrm{H}$ than $\mathrm{C}-\mathrm{O}$ interaction. In contrast, the $\operatorname{CCSD}(\mathrm{T})$ data from ref. 37 yield anisotropies $\delta$ in the interval $[0.6,1.0]$ which is in qualitative agreement with the findings of ref. 43 for water outside a CNT. In passing, we note that the $\operatorname{CCSD}(\mathrm{T})$ data for sites A, B and C (rows \# 7-9 of Table 1) cannot be reproduced by a single LJ model. However, this is believed to be less significant because the differences are well below one $\mathrm{kJ} \mathrm{mol}^{-1}$. This is in coincidence with previous work, where the superlubricity of water flowing through CNTs has been traced back to the smoothness of the inner tube walls. ${ }^{48}$ In fact, in several other simulation studies of water in CNTs the interaction is even integrated over the cylindrical surface, which is equivalent to the assumption of structure-less CNTs. ${ }^{24,49}$

\section{Variation of potentials}

The above fit of the LJ form to the quantum chemical data yields relatively well-defined values for the overall interaction strength $\eta$ and for the range parameters $\sigma_{\mathrm{CO}}$ and $\sigma_{\mathrm{CH}}$, but not for the anisotropy parameter $\delta$, as can be seen from the different $\delta$ values in rows \#6-9 in Table 1 . Thus, to systematically study the effect of anisotropy on the water-carbon 

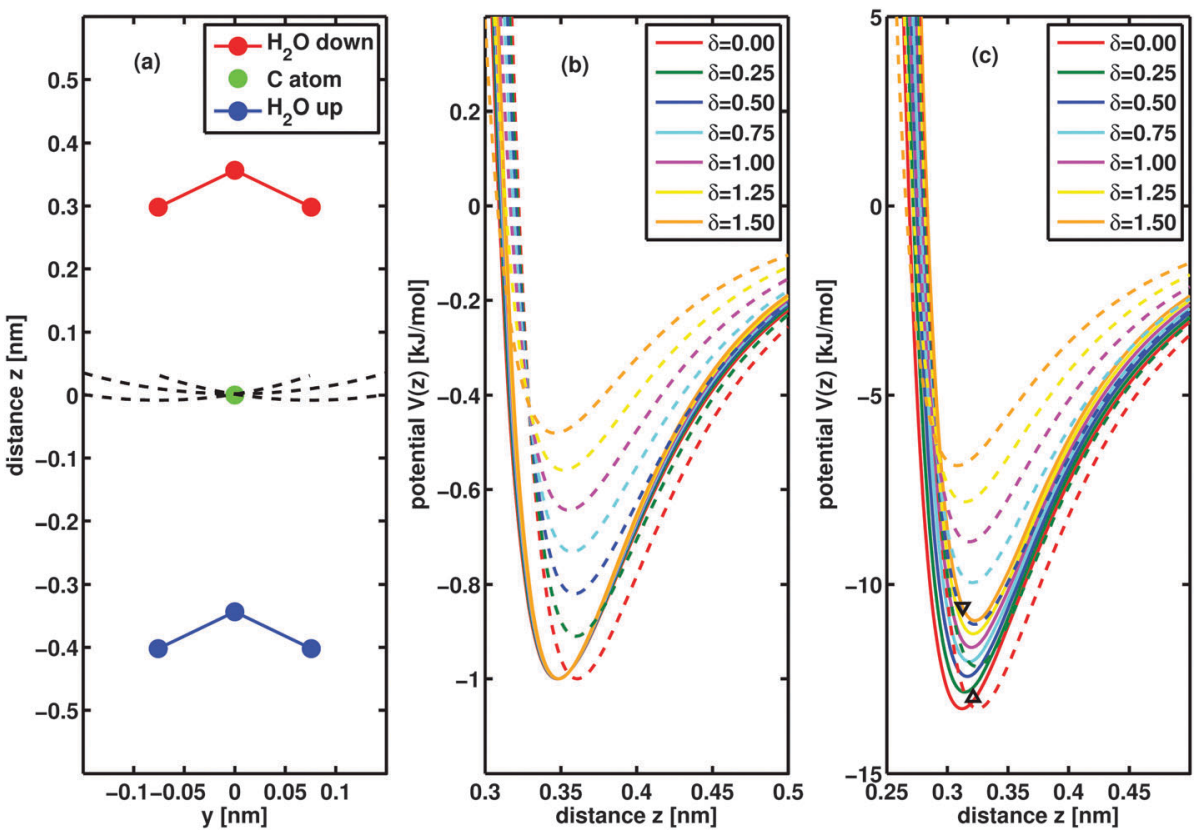

Fig. 1 (a) Down and up orientations of water molecules w.r.t. the carbon position. Radii of circles indicate minimum energy distances $\sqrt[6]{2} \sigma_{\mathrm{CO}}$ and $\sqrt[6]{2} \sigma_{\mathrm{CH}}$ for water down orientation. (b) Pair potentials for water interacting with a single carbon atom, for different values of the interaction anisotropy $\delta=0 \ldots 1.5\left[\mathrm{~kJ}\right.$ mol $\left.{ }^{-1}\right]$ but for the identical value of the overall well depth $\eta=1 \mathrm{~kJ} \mathrm{~mol}^{-1}$. (c) Effective $\amalg$ model potentials for water interacting with the $C_{58}$ graphene model. Solid and dashed curves indicate water down and up orientations, respectively. For comparison, triangles denote the CCSD(T) results from ref. 37.

interaction, we construct a set of model potentials where $\delta$ ranges from the isotropic limit $\delta=0$ up to a value of $\delta=2$ in steps of 0.25 , while keeping $\eta=1 \mathrm{~kJ} \mathrm{~mol}^{-1}, \sigma_{\mathrm{CO}}=0.3157 \mathrm{~nm}$ and $\sigma_{\mathrm{CH}}=0.2726 \mathrm{~nm}$, the values of which appear to vary less, see Table 1. Instead, our emphasis is on the role of the anisotropy $\delta$ which, to the best of our knowledge, has not been explored before.

The resulting set of $\mathrm{LJ}$ parameters are listed in Table 2, and Fig. 1(b) shows the potentials of a water molecule and a single carbon atom as a function of $z$, which denotes the distance between the centers of mass position of the water molecule and the carbon. For the case of water down orientation, the minimum is found at a distance near $z=0.348 \mathrm{~nm}$ and at an energy very close to $\eta=\varepsilon_{\mathrm{CO}}+2 \varepsilon_{\mathrm{CH}}=1 \mathrm{~kJ} \mathrm{~mol}^{-1}$, irrespective of the anisotropy parameter $\delta$. This is because for this water orientation both the $\mathrm{CO}$ and the $\mathrm{CH}$ distances are very close to their respective minimum energy distances $\sqrt[6]{2} \sigma_{\mathrm{CO}}$ and $\sqrt[6]{2} \sigma_{\mathrm{CH}}$, see also the corresponding dashed circles in Fig. 1(a) which intersect almost exactly at the position of the carbon atom. In contrast, the minimum for the water up orientation varies strongly with the anisotropy. Increasing $\delta$ from 0 to 1.5 causes the minimum to be attenuated from $-1 \mathrm{~kJ} \mathrm{~mol}^{-1}$

Table $2 \mathrm{~L}$ well depths $\varepsilon\left(\mathrm{kJ} \mathrm{mol}^{-1}\right)$ for different anisotropy $\delta$ of the carbonwater interaction, as obtained from eqn (3) with the overall interaction strength $\eta$ fixed at $1 \mathrm{~kJ} \mathrm{~mol}^{-1}$

\begin{tabular}{llllllllll}
\hline$\delta$ & 0.00 & 0.25 & 0.50 & 0.75 & 1.00 & 1.25 & 1.50 & 1.75 & 2.00 \\
\hline$\varepsilon_{\mathrm{CO}}$ & 1.000 & 0.875 & 0.750 & 0.625 & 0.500 & 0.375 & 0.250 & 0.125 & 0.000 \\
$\varepsilon_{\mathrm{CH}}$ & 0.000 & 0.062 & 0.125 & 0.188 & 0.250 & 0.312 & 0.375 & 0.438 & 0.500
\end{tabular}

to about $-0.5 \mathrm{~kJ} \mathrm{~mol}^{-1}$. At the same time, the minimum energy distance is reduced from $r=0.361 \mathrm{~nm}$ to $r=0.347 \mathrm{~nm}$ which is a consequence of increasing the $\mathrm{CH}$ attraction while reducing the $\mathrm{CO}$ attraction.

Fig. 1(c) shows the interaction energy between a water molecule and the benchmark system $\mathrm{C}_{58}$. In the isotropic limit $(\delta=0)$, the minimum is found at $V=-13.3 \mathrm{~kJ} \mathrm{~mol}^{-1}$ and at $z=0.312 \mathrm{~nm}($ down $)$ and at $z=0.325 \mathrm{~nm}(u p)$. The effect of the anisotropy is relatively small for the water down orientation, i.e., the water-graphene well depth is reduced to $-11.0 \mathrm{~kJ} \mathrm{~mol}^{-1}$ when going to $\delta=1.5$. However, for the water up orientation, the well depth decreases to $-6.9 \mathrm{~kJ} \mathrm{~mol}^{-1}$ for that value of the anisotropy. Again it is emphasized that quantum chemical results of ref. 37 and 43 for water-graphene suggest that the most realistic values for the anisotropy parameter should be around 0.75 or 1.0 .

\section{Simulation details}

\section{A. Molecular dynamics}

Based on these model potentials with equal overall watercarbon interaction strength $\left(\eta=1 \mathrm{~kJ} \mathrm{~mol}^{-1}\right)$ and for varying anisotropies $\delta$, we study the dynamical behaviour of water inside CNTs. In particular, we choose armchair CNTs with chiral indices $m=n \in\{6,7,8\}$ with a C-C distance of $0.1418 \mathrm{~nm}$. The longitudinal densities $N_{\mathrm{H}_{2} \mathrm{O}} / L_{\mathrm{CNT}}$ of water molecules are based on ref. 15, where the dependence of wetting of CNTs of length $L_{\mathrm{CNT}}=1.351 \mathrm{~nm}$ at temperature $T=300 \mathrm{~K}$ and pressure $P=1 \mathrm{bar}$ was studied as a function of the hydrophilicity parameter $\eta=\varepsilon_{\mathrm{CO}}$ (neglecting, however, the 
Table 3 Simulation details: armchair $(n, n)$ CNTs of radius $R_{\mathrm{CNT}}[\mathrm{nm}]$ and length $L_{\mathrm{CNT}}[\mathrm{nm}]$ comprising $N_{\mathrm{CNT}}$ carbon atoms. Water occupancies $N_{\mathrm{H}_{2} \mathrm{O}}$ are obtained by scaling from ref. 15 . Corresponding densities $\rho_{\mathrm{H}_{2} \mathrm{O}}\left[\mathrm{g} \mathrm{cm}^{-3}\right]$ are based on effective radii $R_{\mathrm{CNT}}-R_{\mathrm{C}}$ where $R_{\mathrm{C}}=0.17 \mathrm{~nm}$ is the van der Waals radius of carbon, see eqn (4) and ref. 29

\begin{tabular}{llllrl}
\hline$n$ & $N_{\mathrm{CNT}}$ & $R_{\mathrm{CNT}}$ & $L_{\mathrm{CNT}}$ & $N_{\mathrm{H}_{2} \mathrm{O}}$ & $\rho_{\mathrm{H}_{2} \mathrm{O}}$ \\
\hline 6 & 1968 & 0.404 & 20.140 & 89 & 0.633 \\
7 & 2296 & 0.472 & 20.140 & 208 & 0.966 \\
8 & 1312 & 0.546 & 10.070 & 161 & 1.015 \\
\hline
\end{tabular}

anisotropy, i.e., $\delta=\varepsilon_{\mathrm{CH}}=0$ ). By systematically increasing the well depth parameter $\varepsilon_{\mathrm{CO}}$, those authors found that the water occupancy of CNTs is rather insensitive to the exact choice of that parameter beyond the threshold around $0.2 \ldots 0.3 \mathrm{~kJ} \mathrm{~mol}^{-1}$. The choice of the number of water molecules $\left(N_{\mathrm{H}_{2} \mathrm{O}}\right)$ is shown in Table 3. These numbers are obtained by longitudinal scaling of the results published in ref. 15, which was corroborated partly by own sample simulations. Note that a possible dependence of $N_{\mathrm{H}_{2} \mathrm{O}}$ on the anisotropy parameter $\delta$ is neglected here, thereby limiting ourselves to $N V T$ ensembles with equal $N$. Corresponding water densities $\rho_{\mathrm{H}_{2} \mathrm{O}}=N_{\mathrm{H}_{2} \mathrm{O}} / V_{\mathrm{H}_{2} \mathrm{O}}$ can be obtained on the basis of the volume available to the water molecules ${ }^{29}$

$$
V_{\mathrm{H}_{2} \mathrm{O}}=\pi L_{\mathrm{CNT}}\left(R_{\mathrm{CNT}}-R_{\mathrm{C}}\right)^{2}
$$

with $R_{\mathrm{C}}=0.17 \mathrm{~nm}$ being the van der Waals radius of carbon. ${ }^{47}$ While still much lower for the $(6,6) \mathrm{CNT}$, Table 3 shows that these densities are already very close to the bulk value $\rho_{\mathrm{H}_{2} \mathrm{O}} \approx$ $1 \mathrm{~g} \mathrm{~cm}^{-3}$ for the $(7,7)$ and $(8,8)$ CNTs.

Initial configurations of the water molecules confined in the CNTs are generated following a random insertion procedure. To this end, the CNTs are divided into $f$ fragments of equal length and a configuration with $N_{\mathrm{H}_{2} \mathrm{O}} / f$ water molecules is randomly generated for one fragment. Note that $f$ is chosen such as to minimize the number of trials of the algorithm to generate a non-overlapping configuration of $N_{\mathrm{H}_{2} \mathrm{O}} / f$ water molecules inside the fragments, typically resulting in $5 \leq f \leq 10$. Subsequently, the generated water configurations are replicated $f$ times along the tube axis, with a random rotation around that axis and inverting randomly chosen fragments.

All reported MD-simulations are carried out using the GROMACS package $e^{33-35}$ within the NVT ensemble, where periodic boundary conditions are applied along the longitudinal CNT axis. The water-water interaction is modelled in terms of the 5 particle model TIP5P. ${ }^{50}$ Although a systematic study of different water interaction models is not the focus of the present work, we also conducted selected simulations using the TIP4P model $^{51}$ for comparison, vide infra. The equations of motion are integrated using the leap-frog algorithm with a timestep of 1 fs. Carbon atoms are fixed at their positions, which is known not to affect the flow at high occupancy of the tube, see e.g. ref. 52, while the SETTLE algorithm is used to constrain the internal coordinates of the water molecules. ${ }^{53}$ The whole system is thermostated at $T=300 \mathrm{~K}$ using the velocity-rescaling thermostat with a coupling constant $\tau=0.2$ ps. ${ }^{54}$ Neighbor searching is carried out using a twin-range approach, where the neighbor list is updated every 10 steps, and the cutoff radii for van der Waals and Coulomb interactions are $0.9 \mathrm{~nm}$. The particle-mesh-Ewald method is used to efficiently calculate the long-range electrostatics. To ensure equilibration the first 500 ps of simulation are discarded, after which a production phase of $20 \mathrm{~ns}$ is run. Prior to the calculation of relevant quantities introduced below, the overall center of mass motion of the water molecules is removed.

\section{B. Trajectory analysis}

As a first attempt to analyze our trajectories, we calculated structural properties routinely studied when simulating liquids, such as radial and angular distribution functions and density profiles. However, these functions do not exhibit any notable dependence on the anisotropy parameter $\delta$, despite the large differences in the potential energy functions, see Fig. 1(b) and (c). Instead we shall consider here dynamical quantities based on the individual center of mass positions of the water molecules confined in CNTs. In the paragraphs below we propose several dynamical quantities which appear to be sensitive to the anisotropy $\delta$ of the water-carbon interaction. Note that all analyses are carried out in accordance with the cylindrical symmetry of the CNTs, i.e., separately for axial and radial degrees of freedom. ${ }^{55}$

In our analysis of the water trajectories inside CNTs, we calculate the (dimensionless) global Lindemann index, which is often used as a measure of thermal disorder to characterize atomic or molecular systems. ${ }^{56}$ It is defined as the relative root mean square fluctuations of interparticle distances $r_{\mathrm{ij}}$, averaged over all pairs i,j of $N$ particles

$$
q=\frac{2}{N(N-1)} \sum_{\mathrm{i}<\mathrm{j}} \frac{\sqrt{\left\langle r_{\mathrm{ij}}{ }^{2}\right\rangle-\left\langle r_{\mathrm{ij}}{ }^{2}\right\rangle}}{\left\langle r_{\mathrm{ij}}\right\rangle}
$$

where the angular brackets denote time averages over trajectories and where the minimum image convention has been used to comply with periodic boundary conditions along the CNT axis. Originally developed for the characterization of melting of crystals, this quantity has also been used, e.g., in studies of atomic $^{57}$ and molecular ${ }^{58}$ clusters, in protein dynamics, ${ }^{59}$ and also of bare carbon nanotubes. ${ }^{60}$ The empirical Lindemann criterion states that a transition from solid-like to liquid-like behaviour ("melting") occurs if that index exceeds a threshold of 0.1-0.15. ${ }^{56}$ Although in more recent work on finite size systems slightly different critical values for the Lindemann criterion were found, ${ }^{57-60}$ this criterion is better suited for the detection of structural transitions than criteria based on fluctuations from time-averaged positions. ${ }^{57}$

Another quantity to analyze the mobility of water molecules in our simulations is the mean square displacement (MSD). It is defined as the distance that particles travel in time $t$ from their initial positions

$$
\left\langle\Delta r^{2}(t)\right\rangle=\frac{1}{N} \sum_{i=1}^{N}\left[r_{i}(t)-r_{i}(0)\right]^{2}
$$



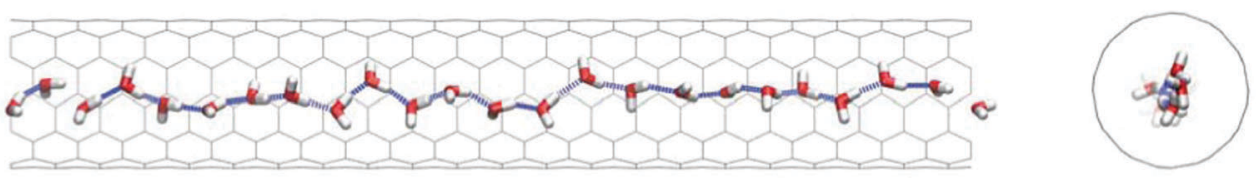

(a)
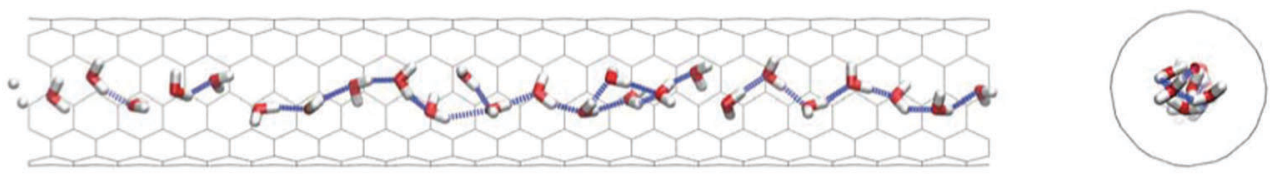

(b)
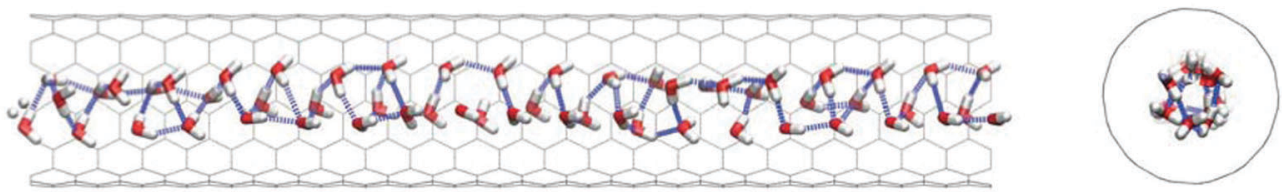

(c)
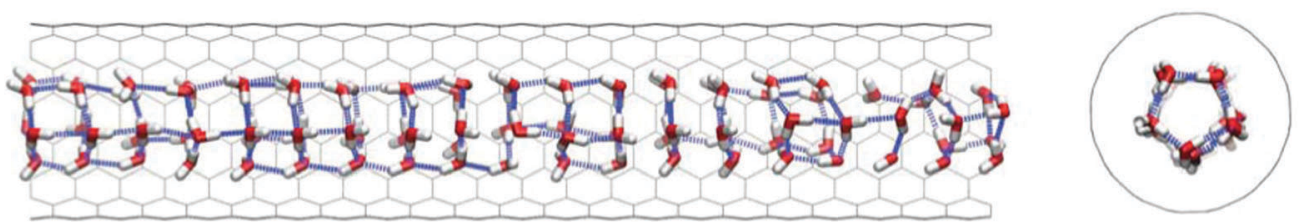

(d)

Fig. 2 Representative snapshots of our $T=300 \mathrm{~K}$ molecular dynamics simulations of water molecules in small CNTs, truncated to a length of $5.5 \mathrm{~nm}$. (a) Water in (6,6) CNTs for $\delta=0.0$ : essentially intact one-dimensional water chains. (b) Water in $(6,6)$ CNTs for $\delta=1.5$ : chain ruptures and formation of compact clusters. (c) Water in $(7,7)$ CNTs for $\delta=1.0$ : strongly perturbed ice nanotubes (three-fold helical). (d) Water in $(8,8)$ CNTs for $\delta=1.0$ : weakly perturbed ice nanotubes (stacked pentagons). Figures generated with the VMD software package. ${ }^{63}$

The slope of the time dependence of the MSD is directly related to the diffusion constant $D$ through the Einstein relation

$$
2 d D=\lim _{t \rightarrow \infty} \frac{\partial\left\langle\Delta r^{2}(t)\right\rangle}{\partial t}
$$

where $d$ stands for the number of spatial dimensions. ${ }^{61}$ As described below, both the Lindemann indices and the MSDs for water molecules can be very different for dynamics along radial and axial directions of the confining CNTs.

We also performed an analysis of H-bonding networks as yet another way to analyze the structures of water in CNTs from our simulations. Being considerably lower than for bulk water, the averaged number of $\mathrm{H}$-bonds has proven to provide valuable insight for water in small CNTs. ${ }^{46}$ Beyond that average, more detailed information is provided by the joint probabilities $p_{n_{\mathrm{a}}, n_{\mathrm{d}}}$ of a water molecule to act $n_{\mathrm{a}}$ times as an acceptor and $n_{\mathrm{d}}$ times as a donor in hydrogen bonding. ${ }^{22,46,62}$ As described below, these probabilities are much more sensitive to changes in the potential anisotropy parameter than the averaged number of H-bonds. To account for the floppy arrangement of water molecules in our simulations for $T=300 \mathrm{~K}$, we use a relaxed criterion for the detection of $\mathrm{H}$-bonds, i.e., $\mathrm{O}-\mathrm{O}$ distances up to
$0.35 \mathrm{~nm}$ and deviation from linearity of the $\mathrm{O}-\mathrm{H}$...O arrangement up to 45 degrees.

As a final tool for analysis we also calculated the decomposition of energies into individual $\mathrm{LJ}$ and Coulomb contributions of the wall-water and the water-water interactions. These quantities, averaged over the molecular dynamics trajectories, can be used as an additional means to learn about energetic and structural changes. A similar idea has been pursued in the characterization of phase transitions of water in CNTs. ${ }^{28}$

\section{Results and discussion}

We present here the results of our MD simulations of water in various armchair CNTs, the sizes of which as well as the respective water occupancies are summarized in Table 3. For the case of the $(6,6)$ and $(7,7)$ tubes, with diameters below $1 \mathrm{~nm}$, we expect the water-CNT interaction to be comparable to the water-water interaction. Hence, the sensitivity of the confined water to variations of the water-carbon potential is worth investigating. We begin our discussion with the case of water inside $(6,6)$ CNTs, which is one of the lowest diameter tubes known to be water-permeable under ambient conditions and 
where all previous simulation studies have found the existence of quasi one-dimensional ordered water chains. We continue by considering $(7,7)$ CNTs, which are the smallest armchair tubes that can accommodate tubular water structures or INTs inside. This section is concluded by reviewing our results for $(8,8)$ CNTs, where the water-water interaction starts to dominate over the water-CNT interaction and where details of the latter are expected to become less important.

\section{A. Water inside $(6,6)$ CNTs}

The simulations of water inside $(6,6)$ CNTs for low to medium values of the anisotropy parameter $(\delta<1.0)$ yield ordered, onedimensional chains of water molecules shown in Fig. 2(a), which were also found in previous simulation work using isotropic interaction models. ${ }^{11,17-20}$ The corresponding analysis in terms of the Lindemann index $q_{\mathrm{a}}$ for the axial motion is shown in Fig. 3(a). For $\delta \leq 1.0$ the Lindemann index $q_{\mathrm{a}}$ is below the critical range of $0.1<q<0.15$, i.e., the water is still solidlike. However, for $\delta \geq 1.25$, there is a steep increase in $q_{\mathrm{a}}$ which indicates the onset of a liquid-like behaviour of water along the $(6,6)$ CNT axis, implying a breakdown of the ordered water chain, see Fig. 2(b). For the radial degree of freedom, however, the Lindemann index $q_{\mathrm{r}}$ is in the liquid-like regime for all $\delta$ values investigated, see Fig. 3(d). Although the absolute variation of $q_{\mathrm{r}}$ with $\delta$ is rather small, there is, however, a distinct increase for the largest $\delta$ value.

Next, the mean squared displacements for center of mass positions of water molecules are investigated, see Fig. 4(a). For $\delta \leq 1$, the axial MSDs have almost no slope indicating the absence of axial self-diffusion, which is still essentially in agreement with the concept of a solid-like one-dimensional ordered water chain. ${ }^{11,17-20}$ In passing, we note that this does not preclude the possibility of transport by concerted water motion through the CNT, which is even known to be extremely fast for the smallest CNTs. ${ }^{11-16}$ However, there is a rapid onset of diffusion (slope of the MSDs) for $\delta \geq 1.25$ which is in contradiction with the notion of ordered, one-dimensional water chains. Instead, the rather large distances travelled by individual water molecules are only possible if water molecules can pass each other within the $(6,6)$ CNT which is indeed confirmed by visual inspection of our trajectories, see Fig. 2(b). This is also supported by the radial MSDs, which do not show a notable drift in time. However, their fluctuations $\left(\sqrt{\left\langle\Delta r_{r}{ }^{2}\right\rangle} \leq 0.07 \ldots 0.1 \mathrm{~nm}\right)$ appear large enough to facilitate passing of the molecules.

Next, we turn our attention to the analysis of the H-bonding networks. The averaged number of $\mathrm{H}$-bonds per molecule shows only little dependence on the anisotropy parameter, decreasing monotonically from $1.944(\delta=0)$ to $1.850(\delta=2)$. The joint probabilities $p_{n_{\mathrm{a}}, n_{\mathrm{d}}}, 2,46,62$ however, do clearly reflect the breaking of the one-dimensional water chains for larger values of $\delta$, see Fig. 5(a). For weakly isotropic models $(\delta<0.75)$, the network is dominated by $p_{1,1} \geq 0.9$, i.e., mainly two-fold coordinated water molecules forming an uninterrupted ordered chain in one dimension, ${ }^{11,17,18}$ see Fig. 1(a).

For larger anisotropy $(\delta \geq 1)$, we find changes in the pattern of H-bonding. First of all, the probability $p_{1,1}$ drops significantly, which is interpreted as evidence for water chain fragmentation. ${ }^{19}$ Two other probability groups show a noticeable rise: those associated with terminal water molecules $\left(p_{1,0}, p_{0,1}\right)$ and those associated with cluster formation $\left(p_{1,2}, p_{2,1}\right)$. Meanwhile, the four-fold coordination dominating the structure of bulk water is practically absent $\left(p_{2,2}<0.01\right)$. The emerging picture is that of a ruptured water chain, where certain parts of the nanotube begin to empty. In other parts of the tube the water molecules cluster into more compact water aggregates, with typical structures
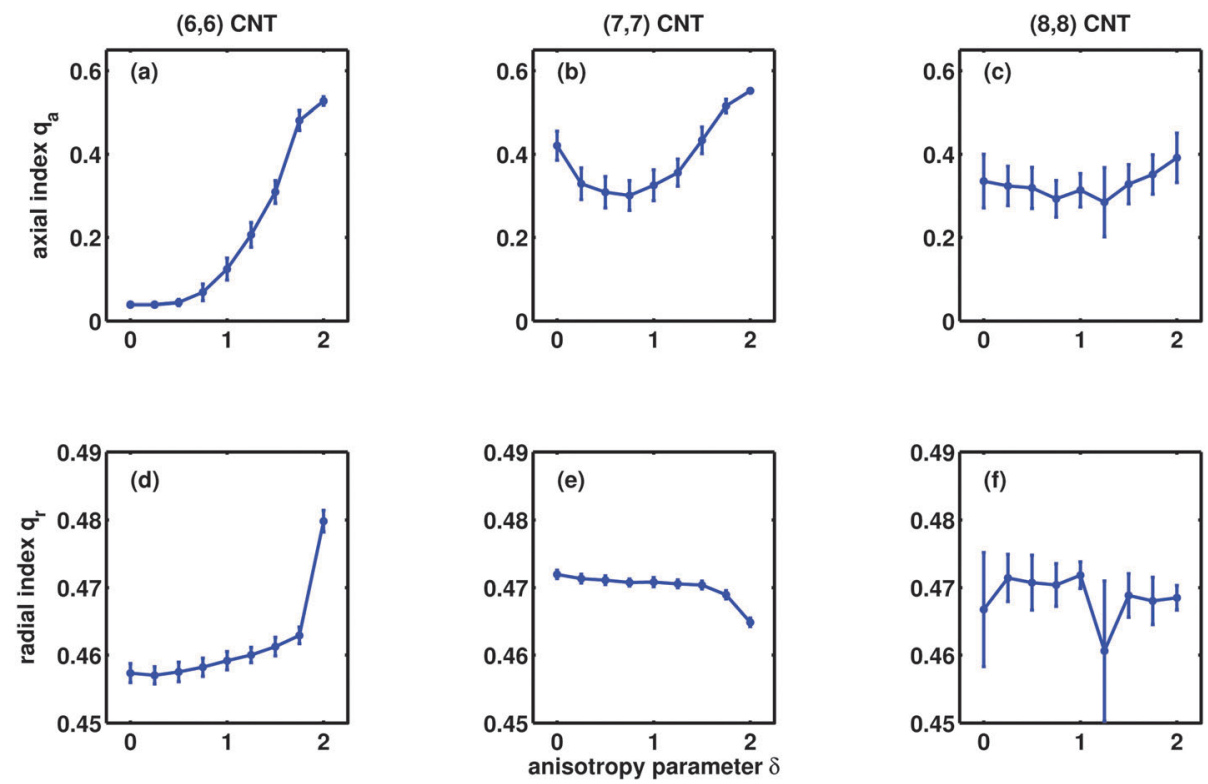

Fig. 3 Lindemann index along axial (upper) and radial (lower) directions, for water molecules inside $(6,6),(7,7)$ and $(8,8)$ armchair CNTs (left to right) for different values of the interaction anisotropy $\delta$. 

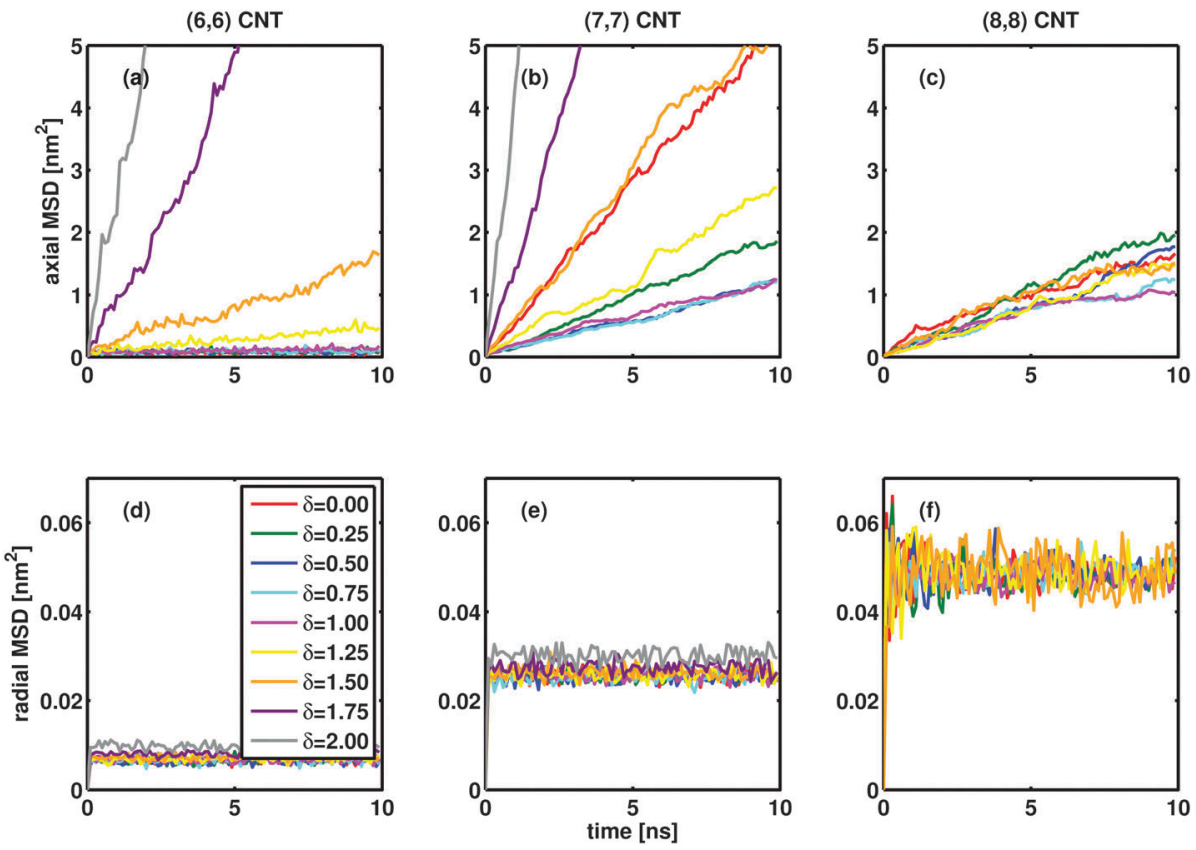

Fig. 4 Mean squared displacements along axial (upper) and radial (lower) directions, for water molecules inside $(6,6),(7,7)$ and $(8,8)$ armchair CNTs (left to right) for different values of the interaction anisotropy $\delta$.
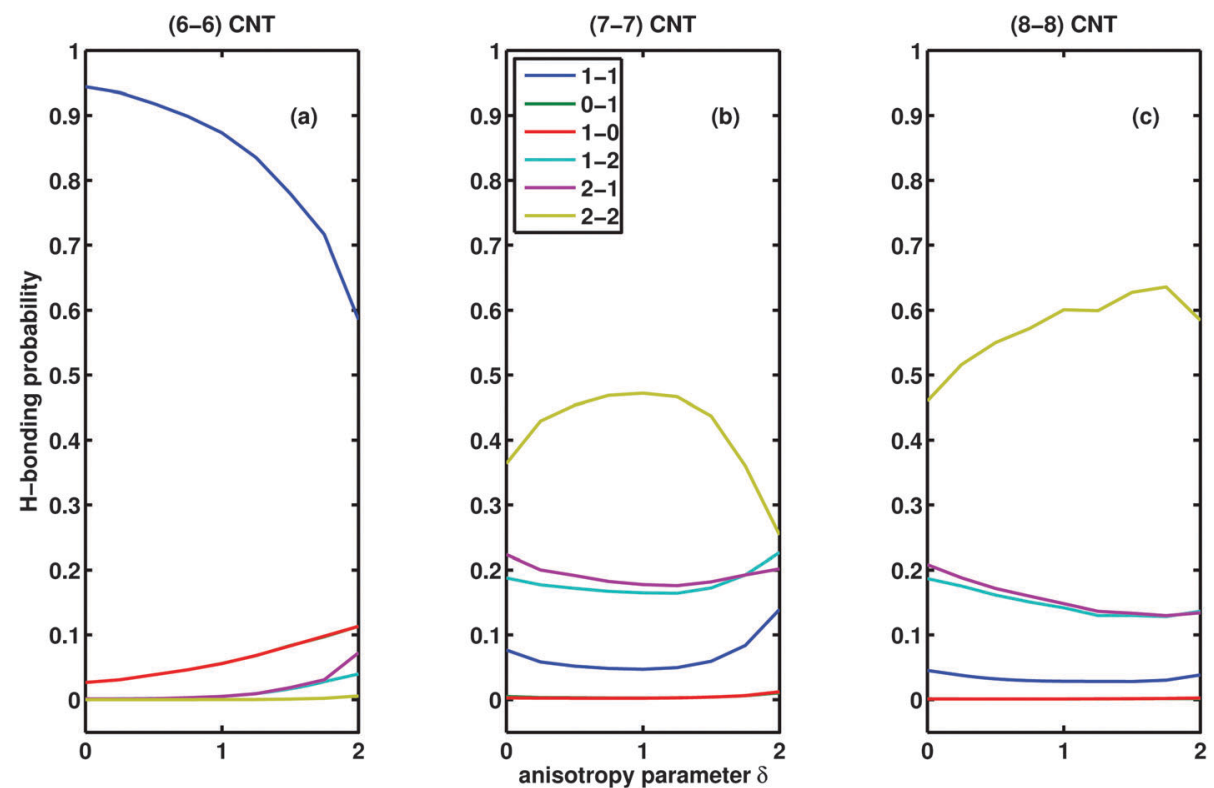

Fig. $5 \mathrm{H}$-bonding patterns of water inside $(6,6),(7,7)$ and $(8,8)$ armchair CNTs (left to right) for different values of the interaction anisotropy $\delta$ given as joint probabilities $p_{n_{a}, n_{d}}$ of a molecule acting $n_{a}$ times as an acceptor and $n_{d}$ times as a donor in a hydrogen bond. Note that green $(0,1)$ and red $(1,0)$ curves practically coincide.

consisting of a chain fragment with extra molecules attached at the side, see Fig. 2(b). This is the key reason for the axial diffusion of water in our simulations with highly anisotropic potentials.

This picture is further supported by the partitioning of the energies (averaged over simulation time and over all water particles). Fig. 6(a) shows that for water in $(6,6)$ CNTs the carbon-water attraction ( $\mathrm{LJ}$ only) is substantially stronger than the water-water interaction (sum of Coulomb and LJ interaction) for the isotropic model $(\delta=0)$. The main energetic effect of increasing the $\delta$ parameter is a drastic reduction of the CNTwater attraction: going from $\delta=0$ to $\delta=2$ destabilizes $E_{\mathrm{CNT}-\mathrm{H}_{2} \mathrm{O}}$ from -34 to $-19 \mathrm{~kJ} \mathrm{~mol}^{-1}$, to the point of being nearly equal to the averaged water-water interaction energy. This destabilization is understood as a consequence of the water clustering and certain molecules coming thereby too close to the CNT walls. 

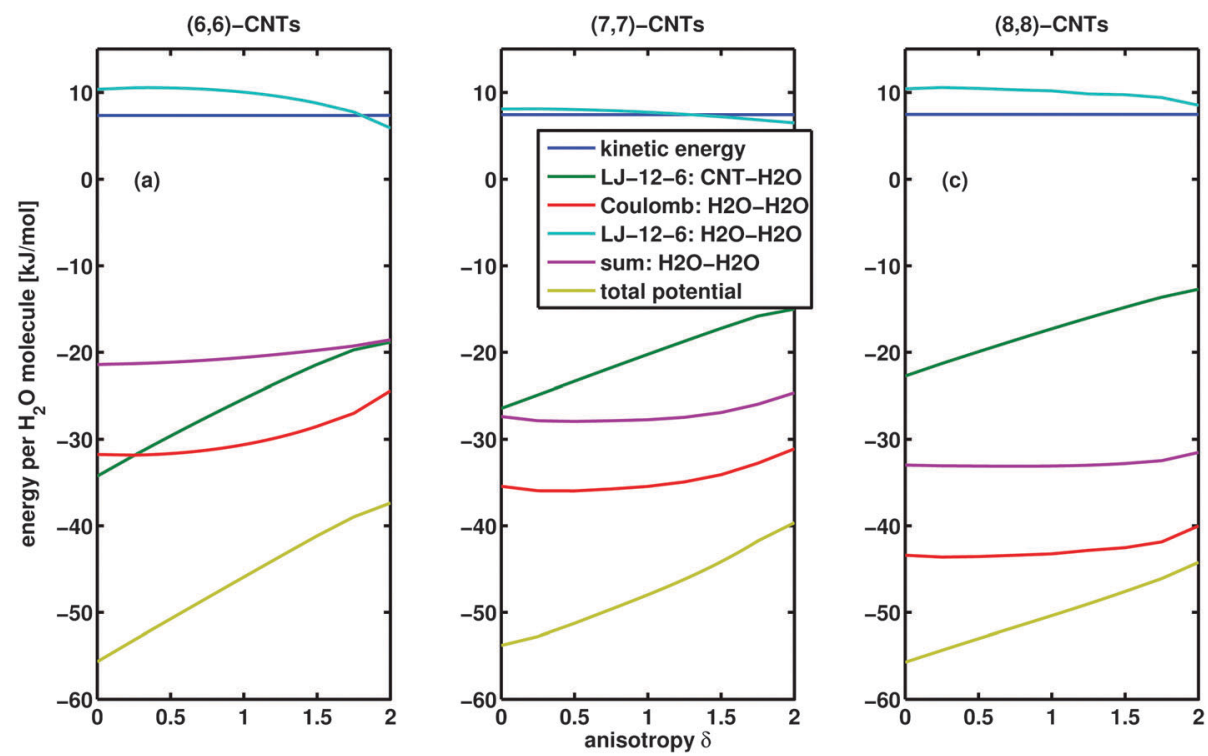

Fig. 6 Decomposition of energies for water molecules inside $(6,6),(7,7)$ and $(8,8)$ armchair CNTs (left to right) for different values of the interaction anisotropy $\delta$.

There is also a trend in the two components of the total $E_{\mathrm{H}_{2} \mathrm{O}-\mathrm{H}_{2} \mathrm{O}}$. While the LJ-contribution is stabilized (indicating less water-water repulsion), the Coulomb attraction is destabilized, although the average number of $\mathrm{H}$-bonds is rather unaffected by increasing $\delta$. That is, a different probability distribution of $p_{n_{\mathrm{a}}, n_{\mathrm{d}}}$ can - while conserving the average value - yield different total values of the water-water interaction energy. In this case, frequent ruptures of the water chains are not sufficiently compensated by the enhanced coordination number of water molecules in other regions of the CNT.

In summary, for these narrow $(6,6)$ CNTs, the overall interaction energy is dominated by the water-nanotube interaction, rather than the water-water interaction which is the reason for the observed anisotropy-dependence of the structural and dynamical properties discussed above. That is also why all our findings are rather insensitive to the choice of the water model: sample simulations with the TIP4P model do not significantly deviate in any of the above analysis. In passing, we also note that for any of the values of $\delta$ considered here, the water-CNT attraction is considerably stronger than the quantum chemical water-graphene energies from ref. 37-42 used in the parametrization of our LJ-parameters, see also Fig. 1, which is a direct consequence of the curvature of the $(6,6)$ CNT walls essentially interacting with the encapsulated water molecules from all directions.

\section{B. Water inside $(7,7)$ CNTs}

Changing from the $(6,6)$ CNTs to the $(7,7)$ armchair CNTs increases the radius from 0.404 to $0.472 \mathrm{~nm}$, thereby increasing $V_{\mathrm{H}_{2} \mathrm{O}}$ by $65 \%$, see eqn (4) and Table 3 . Based on the wetting simulations of ref. 15, we scale $N_{\mathrm{H}_{2} \mathrm{O}}$ up from 89 to 208 .

We start the analysis for moderately anisotropic interaction potentials with $0.75 \leq \delta \leq 1$ for two reasons: it is in the approximate range according to the quantum chemical data of ref. 37 and most of the analyzed properties show an extremum around $\delta=1$, see Fig. 3(b)-6(b). Visual inspection of the trajectories shows that the water structures are close to ice nanotubes (INTs), see the representative snapshot in Fig. 2(c). However, even with our rather tolerant criteria for the definition of H-bonds ( $0.35 \mathrm{~nm}, 45 \mathrm{deg}$ ), only about $50 \%$ of the water molecules are found to be four-fold coordinated, as can be seen in Fig. 5(b). This is a finite temperature effect $(T=300 \mathrm{~K})$, where strong fluctuations and many defects perturb the INT structures inside the carbon tube. Still, we can assign most of the structures for $0.75 \leq \delta \leq 1$ to helical, rather than prismatic, trigonal INT structures, i.e. $(3,1)$ rather than $(3,0)$ INTs in the terminology of ref. 24 . That $\delta=1$ exhibits the most solid-like behaviour (even if perturbed) is reflected by the lowest axial Lindemann index $q_{\text {a }}$ (Fig. 3(b)) as well as by the lowest axial selfdiffusion (Fig. 4(b)). For either lower $(\delta<0.75)$ or higher $(\delta>1)$ values, no qualitative changes of the water structures can be distinguished by observation. However, the analysis shows that the water becomes more liquid-like in the axial direction, with higher Lindemann index $q_{\mathrm{a}}$ and self-diffusion, see Fig. 3(b) and $4(\mathrm{~b})$, respectively. The picture of a reduced order of the water arrangement is also supported by the analysis of the H-bond networks illustrated in Fig. 5(b). Both for low and high anisotropy $\delta$, the probability $p_{2,2}$ of finding four-fold coordinated water molecules decreases substantially, whereas the probability of finding water with three or even two hydrogen bonds increases. We associate this effect with the minor destabilization in the water-water Coulomb energy per molecule seen in Fig. 6(b) for small and large values of $\delta$. However, the main energetic effect is that the water-CNT interaction is either comparable or less stabilizing than the water-water interaction for all anisotropy values, inverting the trend observed for $(6,6)$ CNTs.

In summary, despite considerable thermal disorder occurring in the $T=300 \mathrm{~K}$ simulations, we detected water structures close to three-fold helical INTs. These structures are least affected by 
the CNT-wall if the LJ interaction model is constructed such that the oxygen-carbon pair interaction is slightly stronger than, or equal to, the hydrogen-carbon interaction, i.e. for $0.75 \leq \delta \leq 1$. When keeping the overall interaction strength $\eta$ constant, both an increased oxygen interaction $(\delta \rightarrow 0)$ and an increased hydrogen interaction $(\delta \rightarrow 2)$ tend to exert a torque on the water molecules thereby weakening the network of the $\mathrm{H}$-bonds of the INT-like structures, thus leading to more liquidlike behaviour with axial self-diffusion.

\section{Water inside $(8,8)$ CNTs}

Finally, we analyze our simulations of water in $(8,8)$ CNTs, where $N_{\mathrm{H}_{2} \mathrm{O}}$ was scaled up to 161 , and the tube length shortened to $10 \mathrm{~nm}$ (see Table 3). As the tube diameter increases, the interaction with CNT-walls will have less influence than the water-water interaction. Still, the diameter of the $(8,8)$ CNT is far from where the transition from confined-like to bulk-like behaviour is assumed to take place. ${ }^{10}$

The water molecules are arranged mostly in structures close to non-helical $(5,0)$ INTs consisting of stacked pentagons, ${ }^{24}$ see the representative snapshot in Fig. 2(d). Again, finite temperature effects introduce considerable fluctuations and defects. However, these structural fluctuations appear to occur on larger scales, both in time and in space, than for the $(6,6)$ or $(7,7)$ CNTs. Hence, some of the considered quantities are subject to larger statistical errors, despite our trajectories being as long as 20 ns. Nevertheless, the following picture emerges from the respective right panels of Fig. 3-6: the fluctuating defects of the INT-like structures are enough to yield a value of the axial Lindemann index between 0.3 and 0.4 , which clearly indicates a liquid-like behaviour. However, the bonding pattern of the pentagonal prismatic $(5,0)$ INTs appears to be slightly more rigid than that of the trigonal helical $(3,1)$ INTs inside $(7,7)$ CNTs, as can be seen from the reduced axial self-diffusion and the enhanced probability of four-fold coordination in the H-bonding probabilities, see Fig. 4(c) and 5(c).

The main conclusion of our analysis is that the sensitivity to the anisotropy parameter $\delta$ is considerably less than for the smaller CNTs. Most notably, the axial Lindemann index $q_{\mathrm{a}}$ is within the mentioned statistical errors - practically independent of $\delta$. Similarly, also the differences in the MSDs obtained for different values of $\delta$ are minor, whereas the statistics of the H-bonds still show a trend: $p_{2,2}$ slightly increases with $\delta$. The rationale of why the $\delta$-dependence of the considered quantities almost vanishes can be found in Fig. 6(c). In marked contrast to the situation for $(6,6)$ and $(7,7)$ CNTs, the water-water interaction is dominant over the water-CNT interaction for all values of $\delta$ in the case of $(8,8)$ CNTs. Hence, the water structures are mainly determined by the water model, whereas variations of the water-CNT interaction (such as the anisotropy parameter $\delta)$ have negligible influence. In essence, structure and dynamics of water molecules in $(8,8)$ CNTs are much more stable, due to the formation of pentagonal water structures. Despite an identical number (ideally four) of hydrogen bonds per water molecule, pentagonal INTs are much more stable than trigonal INTs. Indeed, it has been shown that $(5,0)$ INTs are among the most stable INTs inside CNTs, exhibiting a substantially higher melting point. ${ }^{24}$ Similar pentagonal, highly stable arrangements are also found for finite-size clusters encapsulated in CNTs. ${ }^{64}$ There, however, the details of the structures and energies depend on the cluster size. Also for isolated clusters, pentagonal prism-like isomers of ten water molecules, respectively, were found to be particularly stable, although the polarizable TTM2-F model potential was used instead of the TIP5P potential. ${ }^{65}$

\section{Conclusions and outlook}

The starting point for our work on the water-carbon interaction was the existence of high level quantum chemistry results. Despite the generally accepted premise of carbon-based materials being hydrophobic, the calculated water-graphene interaction energy of about $-13 \mathrm{~kJ} \mathrm{~mol}^{-1}$ amounts, after all, to about one half of the water dimerization energy $\left(-28 \mathrm{~kJ} \mathrm{~mol}^{-1}\right.$ for the TIP5P water model or $-25 \mathrm{~kJ} \mathrm{~mol}^{-1}$ for MP2 calculations ${ }^{50}$ ) or about one third of the average potential energy per molecule in room temperature bulk water $\left(-41 \mathrm{~kJ} \mathrm{~mol}^{-1}\right.$ for the TIP5P water model). Furthermore, not only is the interaction non-negligible, but also orientation-dependent. We coded this anisotropy into effective LJ-parameters of the oxygen-carbon and hydrogen-carbon interaction rather than reformulating this widely used pairwise interaction model. Our fit procedure results in an overall potential well $\eta=\varepsilon_{\mathrm{CO}}+2 \varepsilon_{\mathrm{CH}}$ that is notably deeper than those used in most of the previous literature. As already suggested in ref. 43, where similarly strong interaction parameters were determined for water outside CNTs, this may be due to the fact that our effective potentials include not only contributions of dispersion but also polarization interaction. In addition, we find the water-carbon interaction to be strongly anisotropic, a fact which is not considered in most of the simulation literature for water inside CNTs. Although additional quantum-chemical calculations would be desirable to further constrain the anisotropy parameter $\delta=1-\left(\varepsilon_{\mathrm{CO}}-2 \varepsilon_{\mathrm{CH}}\right) / \eta$, high values between 0.75 and 1.0 appear to be most likely. Obviously, there is a need for further quantum chemical investigations to explore not only the orientation dependence of the water-carboninteraction, but also the influence of the curvature of the carbon sheet, which is the main difference between graphene and an actual CNT wall. Ultimately, it should be clarified that, while having the known advantages of a pairwise potential, the reparametrized model carries the same shortcomings intrinsic to all LJ approaches, such as the neglect of polarization and induction effects. Beyond that, the question arises whether a set of universal LJ parameters can be obtained, that is, independent of the curvature of the carbon sheet. Note that it is the curvature of the internal CNT wall that has been proposed as the underlying reason for superlubricity of water flow through CNTs. ${ }^{16}$

Based on this reparametrization of LJ potential models we then carried out a series of MD simulations for TIP5P water in CNTs. In all simulations we use a constant overall well depth parameter $\eta=1 \mathrm{~kJ} \mathrm{~mol}^{-1}$. For the particular case of the lowdiameter tubes studied here, the carbon-water attraction (LJ) is 
approximately equal to (for $(7,7)$ CNTs) or even stronger than (for $(6,6)$ CNTs) the water-water interaction. Hence, the water structures are subject to a subtle interplay between the interaction with the CNT wall and the hydrogen bonding among themselves.

Most importantly, we have shown here for the first time that not only the overall water-carbon interaction strength $\eta$ plays a role, but also the anisotropy of the interaction between the water and the CNT wall $(\delta)$ has to be taken into account for the smaller tubes. Indeed, we find a significant influence on structure and dynamics as well as patterns and energetics of hydrogen bonding of water inside $(6,6)$ and $(7,7)$ CNTs. In our studies of $(6,6)$ CNTs we found qualitatively different behaviour for isotropic and anisotropic models, where the ordered onedimensional chains get ruptured in the latter case, which was not found anywhere in the previous literature to the best of our knowledge. For $(7,7)$ CNTs we found structures that are, although liquid-like, close to helical $(3,1)$ INTs. They are least affected by the attractive interaction with the CNT walls for $0.75 \leq \delta \leq 1$. However, both for lower and higher values of $\delta$, the influence of fluctuations increases, which leads to an even more liquid-like behaviour with notable self-diffusion. However, our findings of anisotropy-dependence of structural, dynamic, and energetic properties are essentially limited to the case of $(6,6)$ and $(7,7)$ CNTs with diameters below $1 \mathrm{~nm}$, where the water-nanotube interaction is equal to, or even stronger than, the water-water interaction. In contrast, for larger CNTs the water-water interaction tends to dominate over the water-wall attraction and details of the water-carbon interaction become less important. This is exemplified in the case of water inside $(8,8)$ CNTs, where the water structures resemble non-helical, pentagonal $(5,0)$ INT structures (known to be particularly stable), and where we find practically no effects of $\delta$-dependence any more. Naturally, the more the water-water interaction dominates the structural and dynamical properties, the more these properties will be affected by the choice of the water model (e.g. SPCE, TIP3P, TIP4P), and less by the anisotropy value. However, a study comprising the variation of both the water model and the watercarbon interaction is beyond the scope of this paper.

Finally, it is emphasized that the construction of our LJ models with parameters $\eta, \delta$ as defined in eqn (2) allows us to continuously adjust the anisotropy $\delta$ while keeping the overall water-carbon interaction strength $\eta$ unchanged. Hence, it could be used for molecular dynamics simulations of water at hydrophobic surfaces where the anisotropy needs to be considered only in the vicinity of the interface while a coarse grained isotropic model is sufficient far from the interface. Similar multi-scale simulation techniques have been used in studies of $\mathrm{H}$-bond networks in the solvation of hydrophobic fullerenes where a continuous switching between spherical and non-spherical representations of the water-water interaction allows for an adjustable resolution. ${ }^{66}$

\section{Acknowledgements}

The authors are grateful to Luigi Delle Site and Beate Paulus for helpful discussions. Financial support through the Center for
Scientific Simulations of the Free University Berlin is acknowledged.

\section{References}

1 A. K. Geim and K. S. Novoselov, Nat. Mater., 2007, 6, 183.

2 S. Iijima, Nature, 1991, 354, 56.

3 R. Saito, G. Dresselhaus and M. S. Dresselhaus, Transport Properties of Carbon Nanotubes. Physical Properties Of Carbon Nanotubes, World Scientific, Singapore, 1998.

4 S. Reich, C. Thomsen and J. Maultzsch, Carbon Nanotubes, Wiley-VCH, 2004.

5 A. Kalra, S. Garde and G. Hummer, Proc. Natl. Acad. Sci. U. S. A., 2003, 100, 10175.

6 F. Zhu and K. Schulten, Biophys. J., 2003, 85, 236.

7 R. R. Meyer, J. Sloan, R. E. Dunin-Borkowski, A. I. Kirkland, M. C. Novotny, S. R. Bailey, J. L. Hutchinson and M. L. H. Green, Science, 2000, 289, 1324.

8 J. K. Holt, H. G. Park, Y. Wang, M. Stadermann, A. B. Artyukhin, C. P. Grigoropoulos, A. Noy and O. Bakajin, Science, 2006, 312, 1034.

9 X. Qin, Q. Yuan, Y. Zhao, S. Xie and Z. Liu, Nano Lett., 2011, 11, 2173.

10 A. Alexiadis and S. Kassinos, Chem. Rev., 2008, 108, 5014.

11 G. Hummer, J. C. Rasaiah and J. P. Noworyta, Nature, 2001, 414, 188.

12 M. Majumder, N. Chopra, R. Andrews and B. J. Hinds, Nature, 2005, 438, 44.

13 S. Joseph and N. R. Aluru, Nano Lett., 2008, 8, 452.

14 J. Thomas and A. McGaughey, Phys. Rev. Lett., 2009, 102, 1. 15 M. Melillo, F. Zhu, M. A. Snyder and J. Mittal, J. Phys. Chem. Lett. , 2011, 2, 2978.

16 D. J. Bonthuis, K. F. Rinne, K. Falk, C. Nadir Kaplan, D. Horinek, A. Nihat Berker, L. Bocquet and R. R. Netz, J. Phys.: Condens. Matter, 2011, 23, 184110.

17 A. Berezhkovskii and G. Hummer, Phys. Rev. Lett., 2002, 89, 064503.

18 C. Dellago, M. Naor and G. Hummer, Phys. Rev. Lett., 2003, 90, 105902.

19 J. Köfinger, G. Hummer and C. Dellago, Proc. Natl. Acad. Sci. U. S. A., 2008, 105, 13218.

20 L. Wang, J. Zhao, F. Li, H. Fang and J. P. Lu, J. Phys. Chem. C, 2009, 113, 5368.

21 W. H. Noon, K. D. Ausman, R. E. Smalley and J. Ma, Chem. Phys. Lett., 2002, 355, 445.

22 J. Wang, Y. Zhu, J. Zhou and X.-H. Lu, Phys. Chem. Chem. Phys., 2004, 6, 829.

23 J. Bai, J. Wang and X. C. Zeng, Proc. Natl. Acad. Sci. U. S. A., 2006, 103, 19664.

24 D. Takaiwa, I. Hatano, K. Koga and H. Tanaka, Proc. Natl. Acad. Sci. U. S. A., 2008, 105, 39.

25 R. J. Mashl, S. Joseph, N. R. Aluru and E. Jakobsson, Nano Lett., 2003, 3, 589.

26 J. Bai, C.-R. Su, R. D. Parra, X. C. Zeng, H. Tanaka, K. Koga and J.-M. Li, J. Chem. Phys., 2003, 118, 3913. 
27 K. Koga, G. T. Gao, H. Tanaka and X. C. Zeng, Nature, 2001, 412, 802.

28 J. Shiomi, T. Kimura and S. Maruyama, J. Phys. Chem. C, 2007, 111, 12188.

29 H. Kyakuno, K. Matsuda, H. Yahiro, Y. Inami, T. Fukuoka, Y. Miyata, K. Yanagi, Y. Maniwa, H. Kataura, T. Saito, M. Yumura and S. Iijima, J. Chem. Phys., 2011, 134, 244501.

30 M. Sadeghi and G. A. Parsafar, J. Phys. Chem. B, 2012, 116, 4943.

31 W. L. Jorgensen and J. Tirado-Rives, J. Am. Chem. Soc., 1988, 110, 1657.

32 W. L. Jorgensen, D. S. Maxwell and J. Tirado-Rives, J. Am. Chem. Soc., 1996, 118, 11225.

33 H. Berendsen, D. van der Spoel and R. van Drunen, Comput. Phys. Commun., 1995, 91, 43.

34 E. Lindahl, B. Hess and D. van der Spoel, J. Mol. Model., 2001, 7, 306.

35 B. Hess, C. Kutzner, D. van Der Spoel and E. Lindahl, J. Chem. Theor. Comput., 2008, 4, 435.

36 T. Werder, J. H. Walther, R. L. Jaffe, T. Halicioglu and P. Koumoutsakos, J. Phys. Chem. B, 2003, 107, 1345.

37 E. Voloshina, D. Usvyat, M. Schütz, Y. Dedkov and B. Paulus, Phys. Chem. Chem. Phys., 2011, 13, 12041.

38 E. M. Cabaleiro-Lago, J. A. Carrazana-García and J. Rodríguez-Otero, J. Chem. Phys., 2009, 130, 234307.

39 G. R. Jenness and K. D. Jordan, J. Phys. Chem. C, 2009, 113, 10242.

40 G. R. Jenness, O. Karalti and K. D. Jordan, Phys. Chem. Chem. Phys., 2010, 12, 6375.

41 M. Rubes, P. Nachtigall, J. Vondrasek and O. Bludský, J. Phys. Chem. C, 2009, 113, 8412.

42 M. Rubes, J. Kysilka, P. Nachtigall and O. Bludský, Phys. Chem. Chem. Phys., 2010, 12, 6438.

43 M. Kaukonen, A. Gulans, P. Havu and E. Kauppinen, J. Comput. Chem., 2012, 33, 652.

44 J. Suarez and F. Huarte-Larranaga, J. Chem. Phys., 2012, 137, 064320.

45 J. H. Walther, R. Jaffe, T. Halicioglu and P. Koumoutsakos, J. Phys. Chem. B, 2001, 105, 9980.
46 M. C. Gordillo and J. Marti, Chem. Phys. Lett., 2000, 329, 341. 47 A. Bondi, J. Phys. Chem., 1964, 68, 441.

48 K. Falk, F. Sedlmeier, L. Joly, R. R. Netz and L. Bocquet, Nano Lett., 2010, 10, 4067.

49 J. Breton, J. Gonzalez-Platas and C. Girardet, J. Chem. Phys., 1994, 101, 3334.

50 M. W. Mahoney and W. L. Jorgensen, J. Chem. Phys., 2000, 112, 8910.

51 W. L. Jorgensen, J. Chandrasekhar, J. D. Madura, R. W. Impey and M. L. Klein, J. Chem. Phys., 1983, 79, 926.

52 A. Striolo, Nano Lett., 2006, 6, 633.

53 S. Miyamoto and P. A. Kollman, J. Comput. Chem., 1992, 13, 952.

54 G. Bussi, D. Donadio and M. Parrinello, J. Chem. Phys., 2007, 126, 014101.

55 B. Schmidt and G. Perez-Hernandez, Metastable 0.5: A MATLAB software package for the analysis of molecular trajectory data, Available via http://sourceforge.net/p/traj lab/metastable, 2012.

56 F. A. Lindemann, Z. Phys., 1911, 11, 609.

57 Y. Zhou, M. Karplus, K. D. Ball and R. S. Berry, J. Chem. Phys., 2002, 116, 2323.

58 U. Buck, B. Schmidt and J. G. Siebers, J. Chem. Phys., 1993, 99, 9428.

59 Y. Zhou, D. Vitkup and M. Karplus, J. Mol. Biol., 1999, 285, 1371.

60 K. Zhang, G. M. Stocks and J. Zhong, Nanotechnology, 2007, 18, 285703.

61 D. Frenkel and B. Smit, Understanding Molecular Simulation, Academic Press, San Diego, 2002.

62 N. Agmon, Acc. Chem. Res., 2012, 45, 63.

63 W. Humphrey, A. Dalke and K. Schulten, J. Mol. Graphics, 1996, 14, 33.

64 J. Hernandez-Rojas, F. Calvo, J. Bretón and J. M. Gomez Llorente, J. Phys. Chem. C, 2012, 116, 17019.

65 B. Bandow and B. Hartke, J. Phys. Chem. A, 2006, 110, 5809.

66 B. P. Lambeth, C. Junghans, K. Kremer, C. Clementi and L. D. Site, J. Chem. Phys., 2010, 133, 221101. 